ISSN: 1130-3743 - e-ISSN: 2386-5660

DOI: http://dx.doi.org/10.14201/teoredu29287108

\title{
EL DESARROLLO DOCENTE DEL PROFESORADO UNIVERSITARIO: ANÁLISIS Y SISTEMATIZACIÓN DEL CONCEPTO ${ }^{1}$
}

\author{
Faculty development of university teachers: analysis \\ and systematization of the concept
}

\section{La formation des enseignants d'université: analyse et systématisation du concept}

\begin{abstract}
Alba Madinabeitia EZKurra e Idoia Fernández Fernández Universidad del País Vasco. Facultad de Educación. Filosofía y Antropología. Departamento de Teoría e Historia de la Educación. Avenida Tolosa, 70. 20018 Donostia-San Sebastián.alba.madinabeitia@ebu.eus; idoia.fernandez@ebu.eus
\end{abstract}

Fecha de recepción: enero de 2017

Fecha de aceptación: marzo de 2017

RESUMEN

El desarrollo docente del profesorado universitario es reconocido como un factor relevante para la mejora de la calidad educativa en las instituciones de educación superior. No obstante, detrás de este término se esconden concepciones y prácticas diferentes. Este trabajo pretende analizar este concepto a lo largo del

1. Este artículo emerge como resultado de la actividad del grupo de investigación consolidado IkasGura (GIU 14/08), financiado por la Universidad del País Vasco/Euskal Herriko Unibertsitatea (UPV/ EHU) y, así mismo, integrante de la Unidad de Formación e Investigación «Educación, Cultura y Sociedad (UFI11/54)» de la UPV/EHU. 
tiempo y comprender profundamente la complejidad de sus significados, para lo cual pondremos la atención en los contextos de producción sociohistórica y en la investigación científica. Con tal objetivo se ha realizado una revisión bibliográfica de la literatura anglosajona más relevante en este ámbito educativo. El análisis permite concluir que se ha ido transitando desde un enfoque orientado a apoyar al profesorado en su desarrollo como docente, profesional y/o académico, hacia otro, enfocado a favorecer procesos de enseñanza-aprendizaje efectivos a nivel micro y macro. Estos desafíos explicitan la necesidad de fomentar visiones más amplias que, más allá del profesorado, fijen la atención en las instituciones, los marcos y las infraestructuras que se dirigen a la enseñanza-aprendizaje.

Palabras clave: análisis conceptual; desarrollo docente; profesor de universidad; desarrollo educacional; cambio organizativo.

\section{SUMMARY}

Faculty development of university teachers is recognized as a relevant factor for improving the quality of education in higher education institutions. However, behind this term different conceptions and practices are hiding. This article aims to analyze this concept over time and to understand deeply the complexity of their meanings, for which we will focus on socio-historical production contexts and on scientific research. To this end, it has been made a bibliographical review of the most relevant Anglo-Saxon literature in this educational field. The analysis allows us to conclude that it has been moving from an approach oriented to supporting teachers in their development as teachers, professionals and/or academics, towards another, focused on promoting effective teaching and learning processes at micro and macro level. These challenges highlight the need to foster broader visions that, beyond teachers, focus on institutions, frameworks, and infrastructures that target teaching and learning.

Key words: concept analysis; faculty development; university professors; educational development; organizational change.

\section{SOMMAIRE}

Le développement du corps professoral des enseignants universitaires est reconnu comme un facteur pertinent pour améliorer la qualité de l'enseignement dans les établissements d'enseignement supérieur. Cependant, derrière ce terme, différentes conceptions et pratiques se cachent. Ce travail vise à analyser ce concept au fil du temps et à comprendre profondément la complexité de ses significations, pour lequel nous concentrerons sur les contextes de production socio-historique et sur la recherche scientifique. À cette fin, nous avons fait une revue bibliographique de la littérature anglo-saxonne la plus pertinente dans ce domaine éducatif. L'analyse nous permet de conclure qu'elle est passée d'une approche orientée vers le soutien des enseignants dans leur développement en tant qu'enseignants, professionnels et/ou 
universitaires, vers une autre, axée sur la promotion des processus d'enseignement et d'apprentissage efficaces au niveau micro et macro. Ces défis mettent en évidence la nécessité de promouvoir des visions plus larges qui, au-delà des enseignants, mettent l'accent sur les institutions, les cadres et les infrastructures qui ciblent l'enseignement et l'apprentissage.

Mots clés: analyse conceptuelle; développement des enseignants; professeur d'université; développement de l'éducation; changement organisationnel.

\section{INTRODUCCIÓN}

Los cambios políticos, socioeconómicos y culturales que se han producido en las últimas décadas han ejercido influencia en la educación superior y han generado demandas que podríamos sintetizar bajo un epígrafe común: la necesidad de mejorar la calidad de la enseñanza y el aprendizaje (Barr y Tagg, 1995; Clifford, 1999; Zimmerman, 2002). Un ejemplo es el proceso de Bolonia (Bologna Declaration, 1999; Eurydice, 2007; Fell, Haines y Denicolo, 2009), una decisión de carácter político que ha funcionado como catalizador de cambios en las estructuras de enseñanza-aprendizaje de las universidades europeas y, al mismo tiempo, ha asentado las bases y avivado el debate sobre qué es ser docente en la Universidad y cuál es la naturaleza del trabajo docente inherente a este cambio multinivel (Norton, Sonnemann y Cherastidtham, 2013).

Las políticas de mejora de la calidad de la educación superior adquieren especial relevancia en la Universidad, que es entendida como un escenario social convulso y cambiante en la sociedad del conocimiento (Barnett, 1994; Hargreaves, 2003; Välimaa y Hoffman, 2008). La mejora de la calidad se impulsa políticamente desde los gobiernos e instancias internacionales y se conjuga en la práctica a través de determinados enfoques como las teorías del aprendizaje sociocultural y constructivista que emergen como insignia del cambio pedagógico (Jonassen, 1999; Kolb y Kolb, 2005; Ramsden, 2003); todo ello en un contexto en el que las nuevas tecnologías han proporcionado oportunidades de acceso al conocimiento nunca antes conocidas, y en el que la diversidad étnica y cultural del alumnado demanda constantemente nuevos entendimientos y habilidades por parte del profesorado.

Estas circunstancias han conducido a las instituciones en general y al personal académico en particular a desarrollar nuevas maneras de apoyar y favorecer el aprendizaje de los y las estudiantes. Son cambios que persiguen enriquecer las experiencias de aprendizaje del alumnado (Hanbury, Prosser y Rickinson, 2008; Knapper, 2003).

Si el objetivo último es mejorar el aprendizaje del alumnado resulta ineludible dirigir la mirada a la enseñanza, entendiendo que existe una relación estrecha, aunque no exclusiva, entre ambas. Esta es la razón de que uno de los ejes de la mejora de la calidad educativa sea el perfeccionamiento, capacitación, actualización y/o formación pedagógica del profesorado universitario. La bibliografía anglosajona, 
que además es la que más extensamente ha atendido esta temática, utiliza varios términos, faculty development, academic development, educational development, a la hora de referirse a este tipo de procesos, pero los significados que se atribuyen a cada uno de ellos se intercambian y entremezclan de manera que no tenemos definiciones estrictas y unívocas que permitan comprender con rigor este fenómeno. En español, el término que en cierta manera recoge estas ideas es el de formación del profesorado universitario, pero en este artículo optaremos por hablar de desarrollo docente entendiendo que, aunque es menos frecuente en esta lengua, tiene un corpus de significado muy amplio en la literatura internacional y su análisis puede arrojar luz sobre la propia terminología y enriquecer el concepto predominante en español.

Hablaremos, por tanto, de desarrollo docente del profesorado universitario pero sin pasar por alto que nos referimos a una amalgama de diseños y praxis que ha ido evolucionando con resultados diferentes según los referentes teóricos, el contexto académico y los recursos empleados para ello (Day, 2005; Norton et al., 2013). El desarrollo docente en la Universidad se presenta, a priori, como una cuestión compleja, pero de suma importancia para la mejora del aprendizaje del alumnado.

Realizar un análisis sistemático del concepto desarrollo docente universitario y arrojar un poco de luz en torno a sus múltiples significados es el objetivo principal de este trabajo. Para ello analizaremos su surgimiento y evolución histórica en los países anglosajones que son los que más tempranamente han manifestado su preocupación por este tema y quienes han delimitado con mayor versatilidad los términos y los conceptos que hoy en día encontramos en este ámbito en lengua inglesa. Para abordar este objeto de estudio hemos estructurado este trabajo en torno a dos grandes interrogantes. En primer lugar, nos preguntamos qué se entiende por desarrollo docente en educación superior y, en consecuencia, se analizarán las diferentes definiciones que dan los autores a este concepto y se sistematizarán los significados que se le atribuyen. En segundo lugar, abordaremos la construcción de estos significados en sus contextos de producción sociohistórica y de investigación científica. Para ello tendremos en cuenta tres ejes de análisis: a) La génesis del desarrollo docente en la Universidad y las primeras configuraciones del concepto; b) La irrupción de la medición del impacto de las estrategias de desarrollo docente del profesorado universitario, poniendo especial atención en los énfasis organizativos e institucionales; y c) el surgimiento del concepto de desarrollo educacional o educational development, una nueva síntesis exploratoria en este ámbito.

\section{EL CONCEPTO DE DESARROLLO DOCENTE DEL PROFESORADO UNIVERSITARIO: POLISEMIAS Y SÍNTESIS}

Un primer acercamiento al análisis de qué se entiende por desarrollo docente no deja lugar a dudas: no existe una comprensión universal del concepto y tampoco contamos con una única interpretación válida al respecto. Es un concepto 
que ha sido desarrollado previamente en otros niveles educativos, en los que se constata la misma polisemia que encontramos en educación superior (GarcíaGómez, 1999; Imbernón, 1994; Marcelo y Vaillant, 2010). En este apartado analizaremos diferentes definiciones del término, así como una propuesta que, ya desde finales de la década de los 90, integraba distintas dimensiones dando lugar a un acercamiento más comprensivo y amplio.

Para llevar a cabo el análisis de las definiciones de desarrollo docente hemos seleccionado un determinado número de contribuciones significativas siguiendo el criterio de procedencia de artículos registrados en bases de datos científicas como ERIC O JSTOR. Además, para observar el desarrollo histórico de este concepto se ha seguido tanto el criterio cronológico, porque nos permite determinar los entendimientos sobre el desarrollo docente desde la década de los 70 hasta la actualidad, como el criterio de citación, de manera que recogemos artículos que tienen una amplia cobertura en la literatura científica (Tabla 1).

Teniendo en cuenta el objetivo de este análisis, las preguntas que han guiado el proceso de revisión de la literatura científica son las siguientes:

- ¿Qué énfasis se observan en el contenido de las publicaciones científicas dedicadas al desarrollo docente universitario?

- ¿Cuáles son las principales características o atributos que configuran el marco de sentido del concepto desarrollo docente?

- ¿Cuáles son las discusiones teóricas y pragmáticas que han nutrido este concepto durante su desarrollo?

Si bien en un primer momento se acomete describir y caracterizar de manera general el concepto desarrollo docente informado por el conjunto documental estudiado, se propone principalmente avanzar hacia una sistematización conceptual que permita comprender su desarrollo tomando como referencia las distintas aportaciones teóricas y pragmáticas que lo han nutrido. Esta sistematización conceptual es la que constituirá, finalmente, una aproximación más integradora y clarificadora de este concepto, premisa fundamental en su necesaria normalización. 
TABLA 1

SECUENCIA CRONOLÓGICA DE DEFINICIONES ORIGINALES SOBRE DD EN LA UNIVERSIDAD. ALGUNAS CONTRIBUCIONES IMPORTANTES DESDE 1975 HASTA LA ACTUALIDAD

\begin{tabular}{|c|c|}
\hline AUTOR & DEFINICIÓN \\
\hline Gaff (1975) & $\begin{array}{l}\text { Faculty development in higher education as a those activities that help } \\
\text { teachers improve their instructional skills, design better curricula, } \\
\text { and/or improve the organizational climate for education. }\end{array}$ \\
\hline Francis (1975) & $\begin{array}{l}\text { Faculty development as a process which seeks to modify the } \\
\text { attitudes, skills, and behavior of faculty members toward } \\
\text { greater competence and effectiveness in meeting student } \\
\text { needs, their own needs, and the needs of the institution. }\end{array}$ \\
\hline Centra (1978) & $\begin{array}{l}\text { Faculty development refers to that broad range of activities that } \\
\text { institutions use to renew or assist faculty in their roles. }\end{array}$ \\
\hline $\begin{array}{l}\text { Sheets y } \\
\text { Schwenk } \\
(1990)\end{array}$ & $\begin{array}{l}\text { Faculty development includes initiatives designed } \\
\text { to improve the performance of faculty members in } \\
\text { teaching, research and administration. }\end{array}$ \\
\hline $\begin{array}{l}\text { Bland et } \\
\text { al. (1990) }\end{array}$ & $\begin{array}{l}\text { Faculty development is a planned program to prepare institutions } \\
\text { and faculty members for their academic roles, including } \\
\text { teaching, research, administration, writing and career. }\end{array}$ \\
\hline Lewis (1996) & $\begin{array}{l}\text { Faculty development had evolved into a more expansive term meant } \\
\text { to encompass three key areas of effort: personal development (self- } \\
\text { reflection, vitality, and growth), instructional development (course } \\
\text { and student-based initiatives), and organizational development } \\
\text { (program, departmental, and institution-wide efforts). }\end{array}$ \\
\hline $\begin{array}{l}\text { Wilkerson e } \\
\text { Irby (1998) }\end{array}$ & $\begin{array}{l}\text { Faculty development as a tool for improving the educational } \\
\text { vitality of our institutions through attention to the competencies } \\
\text { needed by individual teachers and to the institutional } \\
\text { policies required to promote academic excellence. }\end{array}$ \\
\hline Hicks (1999) & $\begin{array}{l}\text { Academic development as the provision of pedagogically } \\
\text { sound and discipline relevant development for academic } \\
\text { staff across the broad spectrum of disciplines present within a } \\
\text { university so as to impact effectively on student learning. }\end{array}$ \\
\hline (Bligh, 2005) & $\begin{array}{l}\text { The principal purpose of faculty development is to improve practice. } \\
\text { We use faculty development to manage change and to develop } \\
\text { strengths and skills as well as organizational capacities and culture. } \\
\text { This is achieved through changes in the ways people think about } \\
\text { what they do and in changes to what they do in their works. }\end{array}$ \\
\hline
\end{tabular}

FUENTE. Elaboración propia. 
Una primera revisión de las definiciones permite constatar que aunque hay mucha diversidad todas tienen un denominador común, es decir, todas pretenden incidir en el profesorado universitario a través de un conjunto de procesos, actividades y técnicas que tengan como resultado final la mejora de la calidad de los procesos de enseñanza-aprendizaje en las instituciones de educación superior (Contreras, 2014; Land, 2004; Macdonald, 2002; McLean, Cilliers y Van Wyk, 2008; Professional and Organizational Network in Higher Education [POD], 2007; Sorcine1li, Austin, Eddy y Beach, 2005). Por tanto, se trata en todos los casos de acciones dirigidas al profesorado entendiendo que así se incide en algo ulterior, el aprendizaje de los y las estudiantes.

Una segunda constatación derivada del análisis de estas definiciones es que los autores parecen estar divididos en cuanto al ámbito de influencia del desarrollo docente y en este sentido se trazan dos énfasis claramente diferenciados: el plano individual y el organizativo y/o institucional.

En efecto, hay autores que centran la atención en el profesorado, es decir, ponen el acento en el profesorado como único objeto de mejora (Sheets y Schwenk, 1990). Se trata de una concepción que aparece en la misma génesis del concepto y que permanece hasta hoy día, dando soporte a numerosas propuestas de desarrollo. Es en cierto modo una idea arquetípica que, por ejemplo, Hubbard y Atkins (1995) resumen cuando hablan de hacer avanzar en cada profesor, individualmente considerado, el conocimiento experto disciplinar junto a determinadas habilidades pedagógicas. Este tipo de definiciones parecen interpretar, en términos generales, que entrenando al profesorado universitario para que sea experto en su disciplina y dotándolo de unos conocimientos pedagógicos básicos, se lograría mejorar la calidad de la enseñanza en la Universidad. Se trata de la concepción de desarrollo docente más extendida y sin duda predominante, pero no la única.

Definiciones presentes ya en la década de los 70 amplían el ámbito de incidencia del desarrollo docente a la esfera institucional, introduciendo importantes matices en el concepto. Un buen ejemplo de ello es la definición de Gaff (1975). Este autor norteamericano señala que el desarrollo docente deberá dirigirse a entrenar determinadas habilidades individuales del profesorado pero da un paso más allá y subraya el interés en un nivel que supera el plano exclusivamente individual docente y apunta a mejorar el diseño del currículum y/o mejorar el clima institucional para la educación. Las definiciones de varios autores y autoras se alinean en esta dirección (Bland, Schmitz, Stritter, Henry y Aluise, 1990; Centra, 1978; Francis, 1975; Hicks, 1999; Lewis, 1996; Steinert, 2000; Wilkerson e Irby, 1998), haciendo hincapié en la mejora de las concepciones, habilidades y prácticas docentes (nivel individual), pero, también, dando visibilidad a una amplia esfera mayor a la individual en la que la que el desarrollo docente debe influir. Esta esfera amplia consiste, entre otras, en mejorar el clima organizativo, la vitalidad y cultura institucional, y, por supuesto, la efectividad del aprendizaje del alumnado a través de las estrategias de desarrollo docente. Se trata de aportaciones que en definitiva están considerando que la mejora de la enseñanza y 
el aprendizaje es algo más que la suma de iniciativas del profesorado, que se necesita ir más allá de lo puramente individual.

Una tercera consideración es la de los autores que al hablar de desarrollo docente consideran al profesorado universitario como personas que desempeñan diferentes roles académicos, además del de la enseñanza. En este caso, se trata de concepciones en las que el apoyo se hace extensivo a todos los roles que el profesorado universitario debe desarrollar, incluyendo enseñanza, investigación, administración, escritura científica y gestión de la carrera profesional. Todas ellas figuras arropadas bajo el término faculty o académico, en una traducción forzada (Boyer, 1990; Brew, 2010; Taylor y Canfield, 2007).

En definitiva, al hablar de desarrollo docente conviene ser conscientes de la necesidad de definirlo como un concepto amplio que integra, al menos, dos ámbitos de desarrollo, el concerniente a las actividades ligadas al desempeño de habilidades docentes, así como a las estrategias de mayor complejidad que involucran a la propia organización en la que conviven las y los docentes universitarios. Bajo este criterio de confluencia, los análisis de ciertos autores han situado el desarrollo docente en un campo prolífico, cuando defienden que las estrategias que le dan forma deben operar desde un enfoque holístico y comprensivo (Brew y Boud, 1996; Gibbs, 2004; Groccia, 2010; Lynn Taylor y Rege Colet, 2010; Steinert et al., 2006; Stes, Clement y Van Petegem, 2007).

No obstante, a finales de los 90 nos encontramos con un intento de establecer con mayor precisión los aspectos presentes en las diferentes definiciones del desarrollo docente, integrándolos en un enfoque más global y complejo. Wilkerson e Irby (1998) proponen un acercamiento a partir de cuatro elementos interrelacionados: (1) desarrollo profesional, esto es, guiar al personal académico para que pueda desenvolverse a lo largo del tiempo en un itinerario laboral y profesional en el que irá promocionando y ganando estatus y experiencia; (2) desarrollo instruccional, enfocado a proporcionar a los docentes las habilidades necesarias (skills) para la mejora de la enseñanza; (3) desarrollo del liderazgo, partiendo de la idea de que el éxito de los programas académicos depende de líderes eficaces y currículums bien diseñados, estos líderes deberán desarrollar habilidades propias de la excelencia académica (scholarship) para poder investigar y evaluar planteamientos educativos eficazmente; y (4) desarrollo organizacional, es decir, empoderar al profesorado para que enfatice su rol de educador, llevando a cabo políticas y procedimientos que reconozcan e impulsen la enseñanza y el aprendizaje, así como el desarrollo a lo largo de la vida.

Para alguien que lea esto desde los contextos académicos cuya lengua vehicular es el español la diversidad terminológica y conceptual resulta llamativa. En estos casos se habla de forma predominante de formación para designar la práctica de capacitación pedagógica o perfeccionamiento docente, y podría equivaler al término instructional development (Feixas, Fernández, Lagos, Quesada y Sabaté, 2013). Los términos equivalentes a instructional, staff, professional, academic, faculty, educational y organizational development están prácticamente ausentes en 
el imaginario de la educación superior española o latinoamericana. No obstante, un análisis de los significados que se han ido atribuyendo a estos términos en inglés puede ser de gran ayuda para ampliar las miras y nombrar con mayor precisión prácticas que a día de hoy se encubren bajo el muy genérico término de formación.

Comprender el origen de esta diversidad terminológica y conceptual que se observa en inglés resulta fundamental para poder operar con rigor con estos conceptos y es por esto que analizar el contexto de producción constituye la clave para dar sentido a esta amalgama conceptual (Gosling, 2001; Lynn Taylor y Rege Colet, 2010; Wilkerson e Irby, 1998). En este sentido, Taylor y Colet (2010) señalan que la presencia de varios términos y conceptos es el resultado gradual de un proceso de construcción de conocimiento generado a través de la práctica. Se trata de contextos de producción local, donde los nuevos entendimientos, enfoques e incluso la propia terminología emergen a resultas de la colaboración entre colegas o con especialistas de la enseñanza y el aprendizaje (developers) y con el único objetivo de lograr experiencias de aprendizaje más eficaces.

En la compleja tarea de consensuar qué palabras definen mejor el desarrollo docente, se van observando tendencias que pueden ser sintetizadas y discutidas en aras de tener una mayor comprensión de este espacio de práctica y de investigación (Ouellett, 2010). En la actualidad parece que lo que subyace a estas diferencias terminológicas son dos grandes tendencias internacionales, circunscritas a maneras diferentes de interpretar el desarrollo docente universitario (Fernández y Márquez, 2014). Mientras una línea de actuación pone el foco de atención en el trabajo de apoyo al docente, faculty o professional development -en el caso de EE. UU.-, y academic staff development -en Australia, Europa o Sudáfrica- (Collett y Davidson, 1997; Hicks, 1999); una segunda pone el acento en articular procesos efectivos de enseñanza-aprendizaje tanto a nivel micro (aula) como a nivel macro (institucional), tendencia que se resume en un nuevo término, educational development, procedente del Reino Unido (Gibbs, 1996, 2013; Johnston, 1997). Retomaremos este último término más adelante, pero antes nos detendremos en las situaciones clave que han influido en la construcción interna del concepto.

\section{LA CONSTRUCCIÓN DE SIGNIFICADOS EN LOS CONTEXTOS DE PRODUCCIÓN SOCIOHISTÓRICA Y DE INVESTIGACIÓN CIENTÍFICA}

Los diferentes términos y significados atribuidos al desarrollo docente no son aleatorios sino producto de dinámicas sociohistóricas, muy relacionadas con necesidades contextuales de las universidades en cada momento (Gosling, Sorcinelli y Chism, 2008), así como de las investigaciones y teorizaciones que se han ido produciendo en el ámbito de la educación superior a lo largo del tiempo. La combinación de estos dos análisis nos aporta un mapa de coordenadas para comprender cómo se han ido construyendo los significados de este término y a qué obedecían en cada momento. 


\subsection{Génesis y primeras configuraciones del concepto: desarrollo instruccional $y$ desarrollo profesional}

En este apartado analizaremos el origen del desarrollo docente en educación superior y veremos cómo sus significados se van atemperando según las necesidades que van surgiendo de manera particular en las universidades norteamericanas que son, en definitiva, el embrión de lo que, posteriormente, ha ido sucediendo en otros países. Observaremos cómo se produce una primera diversificación de significados a través de dos términos, desarrollo instruccional y desarrollo profesional, que permiten hacer una primera delimitación conceptual y práctica.

La primera expresión de una estrategia de desarrollo docente en la vida académica fueron los permisos sabáticos, una modalidad que apareció en las universidades norteamericanas en el siglo XIX (Lewis, 1996) y que se extendió a Canadá, Reino Unido y Australia. Consistía en una liberación para el profesorado de sus obligaciones docentes, con el fin de enriquecer su experiencia y conocimiento disciplinar en otras universidades (Crosby, 1962). Este tipo de estrategias eran viables en el marco de universidades de élite, que formaban a estudiantes de clases altas con un interés genuino por el estudio.

Tras la Segunda Guerra Mundial, las universidades pasan a acoger un número superior de estudiantes y convertirse paulatinamente en universidades de masas, en las que el perfil del alumnado y, con ello, las necesidades docentes cambian radicalmente. En el panorama universitario de la década de los 70, los movimientos estudiantiles norteamericanos encienden la preocupación por una educación de calidad (Gaff y Simpson, 1994). Reclaman la actualización pedagógica docente, un mayor control sobre el currículum y la posibilidad de dar feedback al profesorado sobre la calidad de los estudios que cursaban (Ouellett, 2010). Estas críticas afectan profundamente al arquetipo de académico experto en el conocimiento y al mito de que la buena enseñanza se basaba en el dominio de la disciplina. Los métodos tradicionales de enseñanza se muestran insuficientes para atraer a esta nueva generación de estudiantes. El profesorado debe reinventarse en este nuevo contexto y las estrategias de desarrollo docente parecen ser la herramienta necesaria para ello.

En este momento de eclosión masiva de las universidades de masas el gasto en educación se dispara y los órganos gubernamentales comenzaron a exigir la rendición de cuentas y un mayor control sobre las instituciones de Es (Groccia, 2010). El gasto en educación debía ser proporcional a los buenos resultados académicos (Diamond, 2005), a la vez que crecía en la universidad la presión por atraer nuevos estudiantes y conseguir financiación para estar a la vanguardia de la investigación (Wilkerson e Irby, 1998). Una de las medidas institucionales adoptadas para intentar mejorar esta situación fue la de financiar a las universidades para la creación de unidades y programas de desarrollo docente en educación superior. La ideología subyacente consistía en hacer avanzar en cada profesor, individualmente considerado, el conocimiento experto disciplinar junto a determinadas habilidades pedagógicas (Hubbard y Atkins, 1995). Se ponía la atención en definir 
objetivos de aprendizaje apropiados; rediseñar métodos y estrategias; reconsiderar los aspectos relativos a la evaluación del aprendizaje y del curso en sí, y conseguir un buen alineamiento que favoreciera el aprendizaje (Lynn Taylor y Rege Colet, 2010).

En definitiva, se trata de ideas que podríamos clasificar dentro del desarrollo instruccional, basadas en un primer momento en teorías conductistas que identificaban la mejora de la enseñanza con el entrenamiento de determinadas destrezas o habilidades, posteriormente cognitivistas, poniendo el acento en el cambio de las concepciones y enfoques, y finalmente rotando a planteamientos activos de aprendizaje centrados en el alumnado (Barr y Tagg, 1995; Trigwell, Prosser y Waterhouse, 1999). Procesos similares se sucedieron también en las universidades inglesas, donde aparecieron las primeras publicaciones sobre docencia y se fue extendiendo la importancia de un nuevo grupo de profesionales -los educational developers- que tenían como tarea central apoyar al profesorado en el desarrollo de la "buena docencia" (Gibbs, 2004; Gosling et al., 2008). Dicho de otro modo, su misión era proporcionar la ayuda necesaria para que los docentes universitarios desarrollaran su propio conocimiento experto sobre la disciplina y poder así estructurarlo y organizarlo para hacerlo más accesible al estudiante (Kreber, 2002b). A este respecto, Gibbs (2004) pone de manifiesto que la calidad de las prácticas de enseñanza-aprendizaje no depende únicamente de la iniciativa del profesorado universitario, sino que también depende de las políticas institucionales que las promuevan.

No obstante todo este interés emergente por la docencia de calidad y todo el elenco de orientaciones teóricas y estrategias prácticas de desarrollo docente que se extendieron gradualmente por usA, Australia, Canadá y Reino Unido, el prestigio académico no se concentraba en las buenas prácticas de enseñanza, sino en la investigación y en la producción de artículos científicos. A diferencia de lo que ocurre con los niveles de enseñanza no universitarios, el desarrollo docente en educación superior no cubre todos los significados de desarrollo profesional de las personas académicas. Centra (1978), por poner un ejemplo, señala hacer extensivas las estrategias de apoyo al profesorado universitario a todos los roles que desarrolla, como son enseñanza, investigación, administración, escritura científica y gestión de la carrera profesional. Es decir, el énfasis del desarrollo está puesto no solo en cómo conseguir mejores docentes, sino que en cómo pautar también la carrera profesional en la Universidad. De esta manera, el foco se amplía a un personal académico que debe desenvolver a lo largo del tiempo un itinerario laboral y profesional en el que irá promocionando y ganando estatus y experiencia (Kreber, 2002b).

En este tránsito juega un papel especialmente relevante la aportación de Ernest Boyer (1990) que, haciendo un análisis histórico del devenir de las universidades norteamericanas, critica las visiones que de forma restrictiva enfatizan el rol investigador y despojan de valor a otros roles académicos. Su propuesta integral de qué significa ser académico, dividida en cuatro dimensiones de la excelencia académica (scholarship), constituye una síntesis creativa que deja su huella en un concepto 
de excelencia académica de la enseñanza (scholarship of teaching) que, como veremos en el último apartado del artículo, se ha identificado con los niveles más exigentes en los marcos de calificación británicos y australianos.

\subsection{La irrupción de la medición del impacto de las estrategias de desarrollo docente en educación superior: los énfasis organizativos e institucionales}

Otro elemento clave en la evolución y comprensión del desarrollo docente es la investigación sobre su incidencia real e impacto en el aprendizaje. Hemos señalado ya que la puesta en marcha de las unidades y servicios de desarrollo docente coincide con un momento de crecimiento y expansión, pero también con el cuestionamiento de la financiación de las universidades en todo el mundo. En estas circunstancias la necesidad de investigar sobre la efectividad o el impacto de este tipo de estrategias surge, por una parte, impulsada por la dificultad de mejorar profundamente los procesos de enseñanza-aprendizaje y, por otra, por la dificultad de evidenciar su efectividad (Brew, 2007; Chalmers, 2012; Feixas, Fernández, Lagos y Sabaté, 2015; Gibbs y Coffey, 2004; Kirkpatrick, 1994; Parsons, Hill, Holland y Willis, 2012; Steinert et al., 2006; Stes et al., 2007; Weimer y Lenze, 1997).

Una de las propuestas precursoras para superar esas dificultades llega de la mano de Kirkpatrick en 1994 y se basa en un modelo de evaluación que ayuda a organizar el impacto del desarrollo docente en cuatro niveles de resultados educativos: a. Reacciones (satisfacción), b. Aprendizaje (cambios en las actitudes, habilidades y conocimientos), c. Conducta (cambio en la práctica y transferencia de lo aprendido a la práctica), d. Resultados (cambio en el ámbito institucional, organizativo y en el aprendizaje del alumnado). Mientras que los tres primeros niveles de impacto miden la influencia de las estrategias y programas de desarrollo docente en el individuo, el último nivel da un paso más allá y se sitúa en el cambio organizativo-institucional. Esta propuesta de evaluación multinivel permite evidenciar el impacto de enfoques de desarrollo docente más globales y complejos, como lo es el de Wilkerson e Irby (1998) analizado en el primer apartado.

Otro estudio que marca un punto de inflexión en lo referente a la investigación sobre el impacto global de las estrategias de desarrollo docente del profesorado universitario es el de Gibbs y Coffey (2004). Graham Gibbs es un autor británico de gran relevancia dentro de este ámbito y en este trabajo introduce en la ecuación del análisis no solo el plano individual, sino que también habla de medir el aprendizaje del alumnado y hace extensible el análisis a la institución. Este planteamiento no es baladí, puesto que los resultados apuntan a que para cambiar la Universidad de manera global no parecen ser suficientes las estrategias de cambio de las prácticas individuales docentes. Se necesita ir más allá y considerar niveles que ya aparecen en las propuestas de los estudios de impacto.

Los estudios del impacto refuerzan en cierta manera las definiciones de desarrollo docente que ponían el énfasis no solo en el nivel individual (sea entendido como adiestramiento técnico de habilidades, como procesos de cambio 
de concepciones o enfoques, o incluso como procesos socioconstructivistas), y visibilizan la preocupación por el desarrollo profesional y académico, y transitan a niveles más complejos relacionados con lo organizativo, institucional y cultural. Dicho de otro modo, el estudio sobre el impacto de DD funciona como detonador de una apertura multinivel de cambio para avanzar en la mejora de la calidad de los procesos de enseñanza-aprendizaje.

La formulación de estos diferentes niveles en los que cabe esperar el impacto de las estrategias de desarrollo docente presenta nuevos desafíos tanto prácticos como teóricos ya que explicitan la necesidad de que más allá de convertir a los individuos en expertos docentes es necesario fomentar visiones amplias que fijen la atención en las instituciones, los marcos y las infraestructuras que se dirigen a la enseñanza y el aprendizaje (Ceylan, Fiehn, Paetz, Schworm y Harteis, 2011; Urban y Meister, 2010). Sin lugar a dudas el hecho de situar la evaluación del impacto en estos niveles ha forzado un cuestionamiento del propio concepto y lo ha conducido a problematizar otros significados que van más allá del desarrollo instruccional y del desarrollo profesional. Los autores estaban, pues, preparados para dar un nuevo giro conceptual: el desarrollo educacional.

\subsection{El desarrollo educacional: ¿una nueva síntesis?}

Como venimos señalando, la tensión entre los dos focos con los que operan las universidades en el mundo -de un lado, ayudar al profesorado en su desarrollo como docente, profesional y/o académico, y del otro, vehicular procesos de enseñanza-aprendizaje efectivos a nivel micro y macro- va materializándose de manera diversa en cada contexto. A nivel europeo, por ejemplo, impulsada especialmente en la última década por las políticas de construcción del Espacio Europeo de Educación Superior, la tendencia con mayor fuerza parece inclinarse hacia lo que de manera relativamente novedosa se denomina "desarrollo educacional» o educational development (Gibbs, 1996, 2013; Johnston, 1997). En la eclosión de este concepto, por encima de una acomodación frente a las exigencias de carácter competitivo y de rendición de cuentas hacia las universidades, emergen dos principales ideas de cambio que, a continuación, esbozaremos respectivamente. Por un lado, resituar el aprendizaje en el centro de los procesos de enseñanzaaprendizaje; y, por otro, singularizar la Universidad como una organización que aprende.

A pesar de que la idea de trasladar el foco desde los modelos de enseñanza centrados en el profesorado y su disciplina hacia modelos de aprendizaje centrados en el alumnado goza de una larga trayectoria en la comunidad educativa, el protagonismo que se le ha otorgado durante los últimos años con las nuevas políticas de Es tiene, relativamente, un corto recorrido. Hemos analizado en la génesis del desarrollo docente cómo las estrategias de formación situaron el énfasis en incidir sobre el docente de manera individual, no obstante, una multitud de investigaciones posteriores (Barnett, 1994; Barr y Tagg, 1995; Clifford, 1999; Gibbs, 2013; 
Prosser y Trigwell, 1999; Zimmerman, 2002) fueron desplazando el interés hacia la importancia del aprendizaje del alumnado, como fin último sin el que la enseñanza no tendría sentido.

Si bien es cierto que el modelo centrado en el aprendizaje ha concitado el interés de un gran elenco de autores en este ámbito, paulatinamente y acorde con este modelo educativo, una perspectiva más global e institucional va visibilizándose y legitimándose como una buena vía de avance para las universidades: nos referimos a la caracterización de organizaciones basadas en el conocimiento y el aprendizaje (Senge, 2006). Se trata de una manera de concebir la Universidad como una organización que en tiempos de crisis y cambios prioriza la importancia de construir un entorno de aprendizaje en el que el capital humano y las relaciones formales y funcionales que unen las partes de ese sistema educativo -de esa institución- en un todo coherente son esenciales. Como señala Gairín (2000), esta reordenación del contexto organizativo implica una posición activa por parte de las instituciones, quienes asumirían el compromiso de reflejar en sus planteamientos institucionales y materializar a través de prácticas educativas efectivas los cambios que se pretenden impulsar.

En definitiva, estas dos principales ideas de cambio parecen confluir en una nueva síntesis en el ámbito del desarrollo docente del profesorado universitario. Ahora bien, un análisis exhaustivo de las prácticas reales denota que no siempre expresan lo mismo que los discursos. Gibbs (2004) trata de aclarar estas discordancias provenientes de un contexto, el sistema universitario del Reino Unido, donde el desarrollo educacional tiene una larga tradición. Concluye, así, que los cambios a escala que se necesitaban para enfrentarse con el contexto cambiante no se podían conseguir sumando todos los esfuerzos individuales de los profesores, a pesar de su compromiso y competencia. Lo que se requería era una estrategia institucional coherente que ofreciera un marco de actuación en el cual las correspondientes mejoras en la docencia tuvieran lugar y estuvieran adecuadamente amparadas (Gibbs, 2004). Dicho de otro modo, la suma de pequeños esfuerzos aislados en el profesorado no produce el importante efecto de cambio global, ni da como resultado final una Universidad orientada al aprendizaje.

A este respecto, son múltiples los esfuerzos que se han realizado en las unidades y servicios, organizaciones y redes internacionales para el desarrollo docente, así como los investigadores interesados por este tema para lograr dimensionar mejor la enseñanza y el aprendizaje en la Universidad. Gibbs $(2009,2013)$ recopila de manera sintética los movimientos que se han ido dando en las últimas décadas para transitar de la enseñanza al aprendizaje (Tabla 2). Puede apreciarse que el desarrollo educacional ha ido transitando de visiones simples y poco comprometidas a otras más globales y complejas, a medida que el foco ha ido enfatizando en mayor grado el aprendizaje. 
TABLA 2

SÍNTESIS DE LOS ESFUERZOS PARA DESARROLLAR LA ENSEÑANZA Y EL APRENDIZAJE EN LA UNIVERSIDAD

\begin{tabular}{|c|c|}
\hline FOCO EN LA ENSEÑANZA & FOCO EN EL APRENDIZAJE \\
\hline Foco en el aula & $\begin{array}{l}\text { Foco en el entorno de aprendizaje, } \\
\text { evaluación (assessment) y } \\
\text { características sociales }\end{array}$ \\
\hline $\begin{array}{l}\text { Foco en las profesoras y } \\
\text { profesores individuales }\end{array}$ & $\begin{array}{l}\text { Foco en el liderazgo, equipos, grados, } \\
\text { departamentos, institución como un todo }\end{array}$ \\
\hline Pequeño, simple, componente individual & $\begin{array}{l}\text { Amplio, complejo, componentes } \\
\text { interconectados de forma múltiple } \\
\text { operando a múltiples niveles }\end{array}$ \\
\hline Separados, esfuerzos aislados & Integrados, esfuerzos alineados \\
\hline Tácticas de cambio & Estrategias de cambio \\
\hline Aseguramiento de la calidad & Mejora de la calidad \\
\hline Ateórica & Conceptualizada, teorizada \\
\hline Experiencial & Empírica/basada en evidencias \\
\hline Base psicológica & Base social y cultural \\
\hline Tratamiento no académico (unscholarly) & Tratamiento académico (scholarly) \\
\hline Amateur & Profesional \\
\hline $\begin{array}{l}\text { Organizativamente periférica e } \\
\text { impulsando también otras iniciativas }\end{array}$ & $\begin{array}{l}\text { Parte del equipo central e implicada } \\
\text { en la planificación del cambio }\end{array}$ \\
\hline Foco en la calidad mejorando la práctica & $\begin{array}{l}\text { Orientada en nuevas direcciones } \\
\text { implicando nuevas prácticas }\end{array}$ \\
\hline Ajeno al contexto & Dependiente/sensible al contexto \\
\hline
\end{tabular}

FUENTE: GIBBS (2009, 8-9).

De modo que estamos hablando de una especie de integración que bajo el término desarrollo educacional propone articular procesos de enseñanza-aprendizaje efectivos desde niveles locales hasta niveles globales e institucionales (Badley, 1998; Debowski, 2011; Euler, 2015; Gibbs, 1996; Land, 2004; Lynn Taylor y Rege Colet, 2010) que permitan cambiar la Universidad de manera global. Por lo tanto, el desarrollo educacional tiene que ver con cuestionar desde planteamientos más generales las metas del aprendizaje y la enseñanza en Es, acercando a esta discusión también el carácter del currículum y el contexto político y social de la educación (Gosling, 2001).

En resumen, el desarrollo educacional, por tanto, no solo trata de "apoyar", "promover», "desarrollar» y "mejorar» la enseñanza y el aprendizaje, el currículum y 
la evaluación, sino también de dar un tratamiento académico a la enseñanza superior revisándola, analizándola e investigándola (Badley, 1998). Poner la atención en potenciar procesos de EA efectivos tanto a nivel micro como a nivel global e institucional (Johnston, 1997) supone lograr mejores docentes y, a su vez, pautar la carrera profesional en la Universidad, atendiendo por una parte a todos los roles que deben desempeñar y por otra a los niveles previstos y los requisitos necesarios para promocionar de uno a otro.

\section{DisCUSIÓN Y CONSIDERACIONES FINALES}

El análisis del desarrollo docente a lo largo del tiempo da muestras de que el ámbito académico universitario ha sido y es, a nivel internacional, el escenario de propuestas formativas que tienen diferente repercusión en la mejora de la calidad educativa. Así pues, quisiéramos centrar nuestra discusión en dos énfasis construidos desde lógicas diferentes, pero a la vez complementarias, en la tarea de cimentar universidades más eficientes en el desarrollo del aprendizaje.

El primer aspecto que ha generado un largo debate en el ámbito del desarrollo docente es la cuestión del impacto, ¿cuál es el verdadero efecto de las estrategias de desarrollo?, ¿cómo incidir en los niveles organizativo-institucionales y en el aprendizaje de las y los estudiantes?, y, si es posible medirlo, ¿cómo sistematizarlo? Investigar las estrategias de desarrollo docente desde el punto de vista del impacto ha permitido una comprensión más compleja del concepto en sí mismo ya que rompe con la idea simplista de que la calidad de la formación universitaria es simplemente un problema de enseñanza que atañe al profesorado. Se ha llegado a comprender no sólo que el impacto debe ser medido y constatado, sino que debe evidenciarse a diferentes niveles. Se ha transitado a conceptos más complejos y precisos como es el caso del desarrollo educacional, relacionando sujeto, organización, institución y cultura.

El segundo punto de discusión aborda la preocupación que se viene constatando por el desarrollo profesional y académico a lo largo de la carrera docente en la Universidad y que, finalmente, ha desembocado en los llamados marcos de cualificación docente (Chalmers, 2007). Esto implica, tal y como señaló Boyer (1990), que debemos repensar los roles académicos que debe desempeñar el profesorado universitario si queremos alcanzar los niveles más depurados de calidad educativa en ES. Y qué duda cabe, enfatizar el rol docente pasa por promover la profesionalización del trabajo académico de enseñanza y aprendizaje (Scholarship of Teaching and Learning), lo que a día de hoy supone un desafío clave.

Estas reformulaciones o reinterpretaciones de lo que llamamos formación o desarrollo docente deberían conducir a ir adoptando conceptos, como el de desarrollo educacional, que entienden que la mejora de la calidad pasa por la intervención sinérgica y compleja a distintos niveles y que, en consecuencia, las estrategias a desarrollar por las instituciones de Es deben pensarse, diseñarse y evaluarse desde esta perspectiva holística. No obstante, la realidad es que aún queda mucho 
camino por recorrer. Apoyar la profesionalización de la enseñanza mediante la investigación en clase y la investigación activa permite ofrecer oportunidades para el diálogo crítico y el debate sobre la enseñanza y el aprendizaje en escenarios institucionales y departamentales, así como promover el aprendizaje como vínculo crítico que existe entre la enseñanza, la investigación, la profesionalización y el diálogo (Badley, 1998). Además los marcos de cualificación docente existentes en la actualidad (Chalmers, 2007; Chalmers y Gardiner, 2015; Euler, 2015; Margalef García y Álvarez Méndez, 2005; Tigelaar, Dolmans, Wolfhagen y Van der Vleuten, PM, 2004) deben construirse con criterios encaminados a desarrollar procesos de enseñanza-aprendizaje de calidad, entre los que cuentan integrar la excelencia, la investigación y las actividades profesionales en la docencia y el apoyo al aprendizaje de las y los estudiantes.

A modo de conclusión, cabe señalar que las actividades y acciones dirigidas a desarrollos más globales, institucionales y holísticos de la capacidad de las universidades para enseñar y aprender se encuentran en un estado de desarrollo inicial (Fernández y Márquez, 2014). No obstante, parecen necesarias nuevas formas de pensar y actuar que supondrían integrar todos estos elementos en una estrategia institucional coherente de cambio, que dé lugar a las correspondientes mejoras en la docencia y que permita a las y los docentes desenvolverse como académicos (scholars) o profesionales de la docencia (Bland et al., 1990; Smith, 2001).

\section{REFERENCIAS BIBLIOGRÁFICAS}

BADLEY, G. (1998) Making a case for educational development in times of drift and shift. Quality Assurance in Education, 6 (2), 64-73. https://doi.org/10.1108/09684889810205714.

BARNETT, R. (1994) The limits of competence: Knowledge, higher education and society. Bristol, Open University Press. http://dx.doi.org/10.1080/07294360.2015.1011097.

BARR, R. B. y TAGG, J. (1995) From teaching to learning - A new paradigm for undergraduate education. Change: The Magazine of Higher Learning, 27 (6), 12-26. http://dx.doi.org/ 10.1080/00091383.1995.10544672.

Bland, C. J.; Schmitz, C. C.; Stritter, F. T.; Henrry, R. C. y Aluise, J. J. (1990) Successful faculty in academic medicine. New York, Springer Publishing Company. http://dx.doi. org/10.1097/00001888-200005000-00086.

BuIgH, J. (2005) Faculty development. Medical Education, 39 (2), 120-121. http://dx.doi. org/10.1111/j.1365-2929.2004.02098.x.

Bologna Declaration (1999) Joint Declaration of the European Ministers of Education, Convened in Bologna on the 19th of June 1999. Consultado el 12 de septiembre de 2017. http://www.europa.eu.int/comm/education/index_en.html.

BOYER, E. L. (1990) Scholarship reconsidered: Priorities of the professoriate. New Jersey, Princeton University Press. The Carnegie Foundation for the Advancement of Teaching. http://dx.doi.org/10.2307/40250362.

BREw, A. (2007) Evaluating academic development in a time of perplexity. International Journal for Academic Development,12 (2), 69-72. https://doi.org/10.1080/13601440701604823.

BREw, A. (2010) Transforming academic practice through scholarship. International Journal for Academic Development, 15 (2), 105-116. https://doi.org/10.1080/13601441003737618. 
Brew, A. y Boud, D. (1996) Preparing for new academic roles: An holistic approach to development. The International Journal for Academic Development, 1 (2), 17-25. https://doi. org/10.1080/1360144960010203.

CEnTRA, J. A. (1978) Types of faculty development programs. The Journal of Higher Education, 49 (2), 151-162. https://doi.org/10.2307/1979280.

Ceylan, F.; Fiehn, J., Paetz, N.; Schworm, S. y Harteis, C. (2011) Die auswirkungen des bologna-Prozesses-Eine expertise der hochschuldidaktik, en Nickel, S. (ed.) Der Bologna-Prozess Aus Sicht Der Hochschulforschung. Gütersloh, Bundesministerium fur Bildung und Forschung, 106-122. https://doi.org/10.14361/9783839413227-007.

Chalmers, D. (2007) A review of Australian and international quality systems and indicators of learning and teaching. Carrick Institute for Learning and Teaching in Higher Education, 1 (2), 1-122.

Chalmers, D. (2012) Identification and implementation of the indicators and measures of the impact on teaching preparation programs in higher education (TPP impact). Centre for the Advancement of Teaching and Learning (en línea). Consultado el 4 de abril de 2017. http://www.catl.uwa.edu.au/projects/tpp.

Chalmers, D. y Gardiner, D. (2015) An evaluation framework for identifying the effectiveness and impact of academic teacher development programmes. Studies in Educational Evaluation, 46, 81-91. https://doi.org/10.1016/j.stueduc.2015.02.002.

Clifford, V. A. (1999) The development of autonomous learners in a university setting. Higher Education Research y Development, 18 (1), 115-128. https://doi. org/10.1080/0729436990180109.

ColletT, P. y Davidson, M. (1997) Renegotiating autonomy and accountability: The professional growth of developers in a South African institution. The International Journal for Academic Development, 2 (2), 28-34. https://doi.org/10.1080/1360144970020205.

Crosby, E. W. (1962) The origin and early history of sabbatical leave. AAUP Bulletin, 48 (3), 253-256. https://doi.org/10.2307/40222893.

DAy, C. (2005) Formar docentes: Cómo, cuándo y en qué condiciones aprende el profesorado. Madrid, Narcea Ediciones.

Debowski, S. (2011) Emergent shifts in faculty development. To Improve the Academy: Resources for Faculty, Instructional, and Organizational Development, 30, 306-324.

Diamond, R. M. (2005) The institutional change agency: The expanding role of academic support centers. To Improve the Academy, 23, 24-37. Consultado el 12 de septiembre de 2017. http://citeseerx.ist.psu.edu/viewdoc/download?doi=10.1.1.594.6312\&rep=rep $1 \&$ type $=$ pdf.

Euler, D. (2015) Mejorar las competencias docentes del profesorado universitario es necesario, ipero la innovación sostenible requiere algo más! Educar, 51 (1), 149-165. Consultado el 12 de septiembre de 2017. http://www.redalyc.org/pdf/3421/342133060008. pdf.

EuRYDICE (2007) Focus on the structure of higher education in Europe: National trends in the Bologna Process-2006/07. Brussels, Eurydice European Unit.

Feixas, M.; Fernández, A.; Lagos, P.; Quesada, C. y Sabaté, S. (2013) Factores condicionantes de la transferencia de la formación docente en la universidad: un estudio sobre la transferencia de las competencias docentes. Infancia y Aprendizaje, 36 (3), 401-416. https://doi.org/10.1174/021037013807533034.

Feixas, M.; Fernández, I.; Lagos, P. y Sabaté, S. (2015) Modelos y tendencias en la investigación sobre efectividad, impacto y transferencia de la formación docente en educación 
superior. Educar, 51 (1), 81-107. Consultado el 12 de septiembre de 2017. http://www. redalyc.org/pdf/3421/342133060005.pdf.

Fell, T.; Haines, I. y Denicolo, P. (2009) The Bologna process and beyond: Implications for postgraduate education. London, SRHE Publications.

FernánDEZ, I. y MÁrqueZ, D. (2014) Formación docente ¿̨un concepto en crisis? Situación actual y tendencias emergentes en las universidades del Estado español, en MONEREO, C. (ed.) Enseñando a enseñar en la universidad. Sistemas alternativos de formación del profesorado. Parte 3. Prospectiva: El futuro de la formación del profesorado. Barcelona, Octaedro, 251-292.

Fernández March, A. (2009) La gestión de la formación del profesorado en la Universidad. Teoría de la Educación, 20, 275-312. Consultado el 12 de septiembre de 2017. http:// revistas.usal.es/index.php/1130-3743/article/view/993.

Francis, J. B. (1975) How do we get there from here? Program design for faculty development. The Journal of Higher Education, 46 (6), 719-732. https://doi.org/10.2307/1979064.

GafF, J. G. (1975) Toward faculty renewal. San Francisco, Jossey-Bass.

Gaff, J. G. y Simpson, R. D. (1994) Faculty development in the United States. Innovative Higher Education, 18 (3), 167-176.

Gairín, J. (2000) Cambio de cultura y organizaciones que aprenden. Educar, (27), 31-85. Consultado el 12 de septiembre de 2017. http://www.raco.cat/index.php/educar/ article/viewFile/20734/20574.

GarCía-Gómez, S. (1999) El desarrollo profesional. Análisis de un concepto complejo. Revista de Educación, 318, 175-187.

GiBBS, G. (1996) Supporting educational development within departments. The International Journal for Academic Development, 1 (1), 27-37. https://doi. org/10.1080/1360144960010104.

GiBBS, G. (2004) Mejorar la enseñanza y el aprendizaje universitario mediante estrategias institucionales. Educar, 3 (33), 11-30. Consultado el 12 de septiembre de 2017. http:// www.raco.cat/index.php/educar/article/viewFile/20786/20626.

GiBBS, G. (2009) Developing students as learners-varied phenomena, varied contexts and a developmental trajectory for the whole endeavour. Journal of Learning Development in Higher Education ISSN, 31 (1), 1-12. Consultado el 12 de septiembre de 2017. http://www. aldinhe.ac.uk/ojs/index.php?journal=jldhe\&page=article\&op=view\&path[=30\&path[=14.

GIBBS, G. (2013) Reflections on the changing nature of educational development. International Journal for Academic Development, 18 (1), 4-14. https://doi.org/10.1080/13601 44x.2013.751691.

GiBBS, G. y Coffey, M. (2004) The impact of training of university teachers on their teaching skills, their approach to teaching and the approach to learning of their students. Active Learning in Higher Education, 5 (1), 87-100. https://doi.org/10.1177/1469787404040463.

Gosuing, D. (2001) Educational development units in the UK-what are they doing five years on? International Journal for Academic Development, 6 (1), 74-90. https://doi. org/10.1080/13601440110043039.

Gosling, D.; Sorcinelli, M. D. y Chism, N. (2008) The future of faculty/educational development: An international perspective. Paper presented at the Biennial Meeting of the International Consortium for Educational Development Conference. Salt Lake City, UT.

Groccia, J. (2010) Why faculty development why now, en Saroyan, A. y Frenay, M. (eds.) Building teaching capacities in higher education: A comprehensive international model. Sterling, Stylus Publishing, 1-20. 
Hanbury, A.; Prosser, M. y Rickinson, M. (2008) The differential impact of uk accredited teaching development programmes on academics' approaches to teaching. Studies in Higher Education, 33 (4), 469-483. https://doi.org/10.1080/03075070802211844.

HARGREAVES, A. (2003) Teaching in the knowledge society: Education in the age of insecurity. New York, Teachers College Press.

HicKs, O. (1999) Integration of central and departmental development-reflections from Australian universities. The International Journal for Academic Development, 4 (1), 43-51. https://doi.org/10.1080/1360144990040107.

Hubbard, G. T. y Atkins, S. S. (1995) The professor as a person: The role of faculty wellbeing in faculty development. Innovative Higher Education, 20 (2), 117-128. https:// doi.org/10.1007/bf01189643.

IMBERNón, F. (1994) La formación y el desarrollo profesional del profesorado: hacia una nueva cultura profesional. Barcelona, Graó.

Johnston, S. (1997) Educational development units: Aiming for a balanced approach to supporting teaching. Higher Education Research and Development, 16 (3), 331-342. https:// doi.org/10.1080/0729436970160307.

Jonassen, D. H. (1999) Designing constructivist learning environments. Instructional Design Theories and Models: A New Paradigm of Instructional Theory, 2, 215-239.

Kirkpatrick, D. K. (1994) Evaluating training programs: Four levels. San Francisco, Berrett-koehler.

Knapper, C. (2003) Editorial: Three decades of educational development. International Journal for Academic Development, 8 (1-2), 5-9. https://doi.org/10.1080/13601440420 00277892 .

KolB, A. Y. y KolB, D. A. (2005) Learning styles and learning spaces: Enhancing experiential learning in higher education. Academy of Management Learning y Education, 4 (2), 193-212. https://doi.org/10.5465/amle.2005.17268566.

KreBer, C. (2002) Teaching excellence, teaching expertise, and the scholarship of teaching. Innovative Higher Education, 27 (1), 5-23.

LAND, R. (2004) Educational development: Discourse, identity and practice. United Kingdom, McGraw-Hill Education.

Lewis, K. G. (1996) Faculty development in the United States: A brief history. The International Journal for Academic Development, 1 (2), 26-33. https://doi. org/10.1080/1360144960010204.

Lynn Taylor, K. y Rege Colet, N. (2010) Making the shift from faculty development to educational development, en SAROYAn, A. y Frenay, M. (eds.) Building Teaching Capacities in Higher Education: A Comprehensive International Model. Sterling, Stylus Publishing, 139-167.

MacDonald, R. (2002) Educational development: Research, evaluation and changing practice in higher education, en MacDonald, R. y Wisdom, J. (eds.) Academic and Educational Development: Research, Evaluation and Changing Practice in Higher Education. London, Kogan Page, 3-13.

Marcelo, C. y Vaillant, D. (2010) Desarrollo profesional docente: ¿Cómo se aprende a ensenar? Madrid, Narcea Ediciones.

Margalef García, L. y Álvarez Méndez, J. M. (2005) La formación del profesorado universitario para la innovación en el marco de la integración del Espacio Europeo de Educación Superior. Revista de Educación, (337), 51-70. Consultado el 12 de septiembre de 2017. https://www.researchgate.net/profile/Leonor_Margalef/ 
publication/39213005_La_formacion_del_profesorado_universitario_para_la_innovacion_en_el_marco_de_la_integracion_del_Espacio_Europeo_de_Educacion_Superior/ links/569fca8708ae21a5642719a6.pdf.

MCLEAn, M.; Cilliers, F. y VAN WyK, J. M. (2008) Faculty development: Yesterday, today and tomorrow. Medical Teacher, 30 (6), 555-584. http://dx.doi.org/10.1080/01421590802109834.

Muñoz Martínez, M. y Garay Garay, F. (2015) La investigación como forma de desarrollo profesional docente: Retos y perspectivas. Estudios pedagógicos (Valdivia), 41 (2), 389399. https://doi.org/10.4067/s0718-07052015000200023.

Norton, A.; Sonnemann, J. y Cherastidtham, I. (2013) Taking university teaching seriously. Australia, Grattan Institute. Consultado el 12 de septiembre. https://grattan.edu.au/wpcontent/uploads/2013/07/191_Taking-Teaching-Seriously.pdf.

Ouellett, M. L. (2010) Overview of faculty development, en Gillespie, K. J. y RoberTson, D. L. (eds.) A Guide to Faculty Development. San Francisco, Jossey-Bass, 3-20.

Parsons, D.; Hill, I.; Holland, J. y Willis, D. (2012) Impact of teaching development programmes in higher education. York, HEA Research Series.

Professional and Organizational Network in Higher Education (pod) (2007) What is faculty development? Nederland, POD Network.

Prosser, M. y Trigwell, K. (1999) Understanding learning and teaching: The experience in higher education. Buckingham, SRHE and Open University Press.

RAmsden, P. (2003) Learning to teach in higher education. New York, Routledge. https://doi. org/10.4324/9780203507711.

Senge, P. M. (2006) The fifth discipline: The art and practice of the learning organization. New York, Broadway Business.

SheETS, K. J. y SCHwenk, T. L. (1990) Faculty development for family medicine educators: An agenda for future activities. Teaching and Learning in Medicine: An International Journal, 2 (3), 141-148. https://doi.org/10.1080/10401339009539447.

Smith, M. (2001) Reforming teacher education: Competing agendas. Journal of Teacher Education, 52 (4), 263-263. Consultado el 12 de septiembre de 2017. http://journals. sagepub.com/doi/pdf/10.1177/0022487101052004001.

Sorcinelli, M. D.; Austin, A. E.; Eddy, P. L. y Beach, A. L. (2005) Creating the future of faculty development: Learning from the past, understanding the present. Bolton, Anker Publishing Company.

SteineRT, Y. (2000) Faculty development in the new millennium: Key challenges and future directions. Medical Teacher, 22 (1), 44-50. https://doi.org/10.1080/01421590078814.

Steinert, Y.; Mann, K.; Centeno, A.; Dolmans, D.; Spencer, J.; Gelula, M. y Prideaux, D. (2006) A systematic review of faculty development initiatives designed to improve teaching effectiveness in medical education: BEME guide n. ${ }^{\circ}$ 8. Medical Teacher, 28 (6), 497-526. https://doi.org/10.1080/01421590600902976.

Stes, A.; Clement, M. y Van Petegem, P. (2007) The effectiveness of a faculty training programme: Long-term and institutional impact. International Journal for Academic Development, 12 (2), 99-109. https://doi.org/10.1080/13601440701604898.

TAYLOR, R. y CANFIELD, P. (2007) Learning to be a scholarly teaching faculty: Cultural change through shared leadership. Transforming a University: The Scholarship of Teaching and Learning in Practice, 21 (3), 233-247. Consultado el 12 de septiembre de 2017. https:// ses.library.usyd.edu.au/bitstream/2123/2134/1/TransUniTaylor21.pdf.

Tigelaar, D. E.; Dolmans, D. H.; Wolfhagen, I. H. y Van Der Vleuten, P. M. (2004) The development and validation of a framework for teaching competencies in higher education. 
Higher Education, 48 (2), 253-268. https://doi.org/10.1023/b:high.0000034318.74275. e4.

Trigwell, K.; Prosser, M. y Waterhouse, F. (1999) Relations between teachers' approaches to teaching and students' approaches to learning. Higher Education, 37 (1), 57-70.

Urban, D. y Meister, D. M. (2010) Strategien der professionalisierung in der hochschuldidaktik. München, Zeitschrift Für Hochschulentwicklung. https://doi.org/10.3217/ zfhe-5-04/10.

VÄlimaA, J. y Hoffman, D. (2008) Knowledge society discourse and higher education. Higher Education, 56 (3), 265-285. https://doi.org/10.1007/s10734-008-9123-7.

Weimer, M. y Lenze, L. F. (1997) Instructional interventions: A review of the literature on efforts to improve instruction. Effective Teaching in Higher Education: Research and Practice, 205-240.

Wilkerson, L. e Irby, D. M. (1998) Strategies for improving teaching practices: A comprehensive approach to faculty development. Academic Medicine, 73 (4), 387-396. Consultado el 12 de septiembre de 2017. http://journals.lww.com/academicmedicine/ Abstract/1998/04000/Strategies_for_improving_teaching_practices_a.11.aspx.

Zimmerman, B. J. (2002) Becoming a self-regulated learner: An overview. Theory into Practice, 41 (2), 64-70. https://doi.org/10.1207/s15430421tip4102_2. 